\title{
Ontogenetic change in relative performance of allozyme genotypes influences detection of heterosis in the earthworm Eisenia andrei
}

\author{
TC McElroy ${ }^{1}$ and WJ Diehl ${ }^{2}$ \\ ${ }^{1}$ Department of Forest Products, Mississippi State University, Mississippi State, MS 39762, USA; ${ }^{2}$ Department of Biological Sciences, \\ Mississippi State University, Mississippi State, MS 39762, USA
}

\begin{abstract}
The effect of ontogeny on relationships between allozyme genotypes and fresh weight was measured weekly throughout the life history of the earthworm Eisenia andrei to test the hypothesis that there is an ontogenetic component to variation in such relationships. Two of six allozyme loci showed a significant increase in apparent heterosis with ontogeny, while one locus showed a significant decrease in apparent heterosis. Three loci showed a significant decrease in the performance of common homozygotes with ontogeny. Patterns of relative genotypic performance varied among loci, but the cumulative effect was an increase in apparent allozyme heterosis later in ontogeny coinciding with a series of positive relationships between multilocus heterozygosity and fresh weight. The results could not be used to determine
\end{abstract}

whether these patterns were caused by selection acting on the loci directly or on loci tightly linked to allozyme loci. However, because the same individuals were used throughout this study and thus allele frequencies and heterozygote deficiency were constant, the presence of both ontogenetic effects and differences in such patterns among loci is not compatible with a general inbreeding effect. Examining relative genotypic performance repetitively using the same individuals through ontogeny or in different environments is a very powerful experimental design for testing the effects of inbreeding or other populational factors.

Heredity (2005) 94, 258-263. doi:10.1038/sj.hdy.6800610

Published online 3 November 2004

Keywords: allozymes; ontogeny; Eisenia andrei; earthworm; heterosis; potence

\section{Introduction}

Heterozygosity-fitness correlations have been predicted and tested with a variety of mathematical models and empirical experimentation in both natural and laboratory settings. The generality of these relationships has been difficult to assess (Britten, 1996; David, 1998). Three hypotheses have prevailed to explain significant correlations between heterozygosity and fitness: (1) the direct effect hypothesis (allozyme loci are target loci), (2) the local effect hypothesis (allozyme loci are linked to target loci), and (3) the general effect hypothesis (allozyme loci reflect the level of genome-wide inbreeding) (reviewed by Hansson and Westerberg, 2002). Evaluation of mechanisms that may cause heterozygosity-fitness relationships has proven difficult because of overlapping predictions and because these relationships have not always been found when expected (Booth et al, 1990; Whitlock, 1993; Mulvey et al, 1994).

Sufficient repeatability of the multilocus heterozygosity (MLH)-growth rate relationship has not been demonstrated in either laboratory or natural populations. Diehl and Koehn (1985) proposed that a reversal in the MLH-growth correlation in a population of Mytilus

Correspondence: WJ Diehl, Department of Biological Sciences, Mississippi State University, PO Drawer GY, Mississippi State, MS 39762, USA. E-mail: wdiehl@biology.msstate.edu

Received 10 October 2003; accepted 13 September 2004; published online 3 November 2004 edulis sampled several months apart was caused by the selective mortality of homozygous individuals early in their life history. David and Jarne (1997) reported variation in heterozygosity-fitness relationships among cohorts in the bivalve Sipsula ovalis. Similarly, Pecon Slattery et al (1993) reported a significant relationship between heterozygosity and age class in the hard clam Mercenaria mercenaria, but the relationship was not consistent over a 3-year sampling period. Consistency of MLH-fitness relationships has also been lacking from a series of studies on Eisenia andrei (Diehl, 1988; Audo and Diehl, 1995; McElroy et al, 1999; McElroy and Diehl, 2001), although some of this inconsistency disappears when a common set of loci are used (Audo and Diehl, 1995). In the above studies, inbreeding, migration, selective mortality, and ontogenetic effects may have affected heterozygosity-fitness relationships.

Few studies have examined the effects of ontogeny on heterozygosity-fitness relationships in the absence of confounding influences of migration, mortality, and the use of different individuals during ontogeny. We tested the hypothesis that there is an ontogenetic component to variation in relative genotypic performance by examining relationships between allozyme genotypes and fresh weight weekly throughout the life history of the earthworm E. andrei. We also tested the specific hypothesis that there is an ontogenetic component to variation in heterosis, and that the ontogenetic pattern varies among allozyme loci. Differences in patterns of heterosis may indicate different roles of allozyme or linked loci at 
various times in the life history of E. andrei. Because the same individuals are used throughout this study, the presence of either ontogenetic effects or differences in such patterns among loci would be very difficult to reconcile with a general inbreeding effect.

\section{Materials and methods}

The earthworms used in these analyses were a subset of an experimental population that has been described previously (Presley et al, 1996; McElroy et al, 1999). We used $E$. andrei from three soil moisture/temperature treatments $\left(3 \mathrm{ml} \mathrm{H} \mathrm{H}_{2} \mathrm{O} / \mathrm{g}\right.$ dry peat moss, $15^{\circ} \mathrm{C}(n=64)$; $\left.3 \mathrm{ml} / \mathrm{g}, 20^{\circ} \mathrm{C}(n=87) ; 4 \mathrm{ml} / \mathrm{g}, 15^{\circ} \mathrm{C}(n=99)\right)$ that promoted similar growth curves (Figure 1) and had sufficient survivorship for the analyses reported here. Earthworms in excluded treatments survived less than 45 weeks. In the absence of significant environmental effects (see below), environments were treated as replicates.

Individual earthworms were raised in separate $25 \mathrm{ml}$ plastic vials with damp peat moss and food. Neutral $\mathrm{pH}$ was maintained by the addition of $\mathrm{CaCO}_{3}(5 \% \mathrm{w} / \mathrm{w})$. Commercial earthworm food (Magic Products Inc., Amherst Junction, WI, USA) mixed with soy-protein baby formula was provided to the earthworms ad libitum. Soil moisture was maintained at nominal levels by wrapping the individual vials in aluminum foil to prevent water loss. Temperature was maintained in several Freas 818 Precision Scientific incubators. Fresh weight was recorded every 2 weeks for each treatment until asymptotic growth was reached at 50 weeks. On each sampling date, earthworms were removed from their vials, rinsed in aged tap water, blotted dry, weighed, and returned to the vial with fresh peat moss and food. Fresh weight is a good surrogate for fitness in this species (Presley et al, 1996).

Allozyme data were collected for glycerol-3-phosphate dehydrogenase (Gpd2; EC 1.1.1.8), malate dehydrogenase (Mdh; EC 1.1.1.37), alanyl amino peptidase (Aap; EC 3.4.1.-), phosphoglucomutase (Pgm; EC 5.4.2.2), mannose phosphate isomerase (Mpi; EC 5.3.1.8), and 6-phosphogluconate dehydrogenase ( $P g d$; EC 1.1.1.44). Polymorphisms were detected with horizontal starch gel

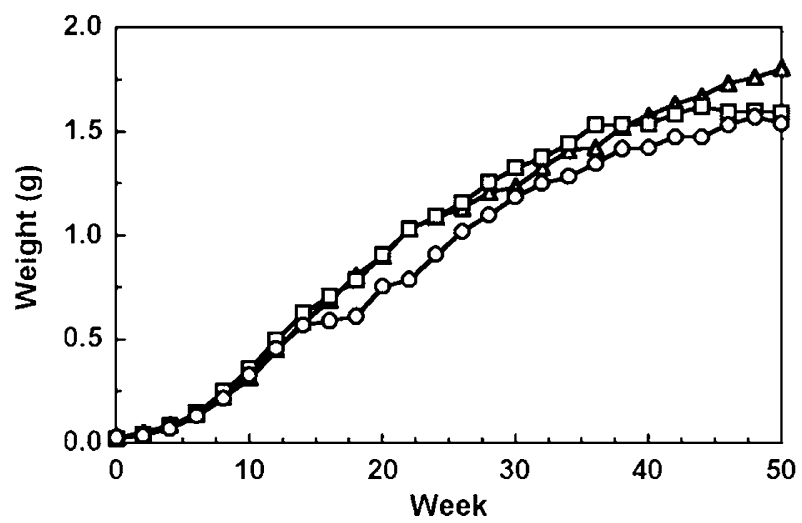

Figure 1 Growth trajectories of E. andrei from each of the three soil moisture/temperature treatments that were used in this study (circles: $3 \mathrm{ml} \mathrm{H} 2 \mathrm{O} / \mathrm{g}$ dry peat moss, $15^{\circ} \mathrm{C}$; squares: $3 \mathrm{ml} / \mathrm{g}, 20^{\circ} \mathrm{C}$; triangles: $4 \mathrm{ml} / \mathrm{g}, 15^{\circ} \mathrm{C}$ ). electrophoresis. Tissues were prepared according to Diehl (1988). Tissue from the posterior end of the earthworm (Diehl, 1988) was homogenized in $100 \mu \mathrm{l}$ of grinding buffer (0.05 Tris- $\mathrm{HCl}$ ( $\mathrm{pH} 8.0$ ) with $20 \%$ glycerol and $0.1 \% \quad \beta$-mercaptoethanol; Diehl, 1988). The homogenate was sonicated to burst the cells and centrifuged at $10000 \mathrm{~g}$ for $15 \mathrm{~min}$. The supernatant was the source of the enzymes.

The gene products of Gpd2 and Mdh were resolved on an amine-citrate buffer system (Clayton and Tretiak, 1972). The gene products of Aap were resolved on a Trisborate-EDTA buffer system (Pasteur et al, 1988). The gene products of $P g m, M p i$, and $P g d$ were resolved on a Trismaleate buffer system (Selander et al, 1971). Enzymes were stained according to Diehl and Williams (1992) with the exception of Aap, which was stained according to Garton et al (1984). All loci except Aap segregated for two alleles creating three genotypes per locus. Aap segregated for four alleles, but individuals with one of the two rare alleles were excluded from these analyses because of incomplete representation of rare genotypes. Only earthworms with complete growth and genetic data were used in the analyses $(N=250)$.

We examined two aspects of genotypic performance: (1) the performance of the heterozygote relative to the respective homozygotes (heterosis) and (2) the performance of the common homozygote relative to the uncommon homozygote. The fitness of heterozygotes relative to homozygous genotypes at single locus was measured as a modification of the potence index of Griffing (1990) for each allozyme locus at each sample date and was calculated as follows:

$$
\text { Het }_{\text {Pot }}=\left[2 F-\left(P_{1}+P_{2}\right)\right] /\left(\left|P_{1}-P_{2}\right|\right)
$$

where $F$ is the fresh weight of the heterozygote, $P_{1}$ the fresh weight of the common homozygote, and $P_{2}$ the fresh weight of the uncommon homozygote. For Het Pot $_{\text {Pon }}$ greater than 1 and less than -1 , the potence index was adjusted as $2-\left(1 /\left|\operatorname{Het}_{\text {Pot }}\right|\right)$ and $-2+\left(1 /\left|\operatorname{Het}_{\text {Pot }}\right|\right)$, respectively. If $\left(\left|P_{1}-P_{2}\right|\right)=0$, then Het $_{\text {Pot }}$ was assigned a value of 2 or -2 as appropriate. Although this adjustment sets upper and lower limits on the data, it had the effect of normalizing the data by reducing kurtosis. The value of Het $_{\text {Pot }}$ can suggest the phenotypic interaction displayed by the locus. If $\operatorname{Het}_{\mathrm{Pot}}=0$, the locus shows codominance. If $\mathrm{Het}_{\mathrm{Pot}}=1$ or -1 , the locus shows complete dominance. If $1 \geq \operatorname{Het}_{\mathrm{Pot}} \geq-1$ and $\operatorname{Het}_{\mathrm{Pot}} \neq 0$, the locus shows incomplete dominance. If $\operatorname{Het}_{\mathrm{Pot}}>1$ or Het $_{\mathrm{Pot}}<-1$, the locus shows overdominance or underdominance, respectively. Heterosis is clearly indicated when Het $_{\text {Pot }}>1$ and is often assumed when Het $_{\text {Pot }}>0$. To determine multilocus effects, we averaged Het $_{\text {Pot }}$ for all loci in each environment at 2-week intervals to generate a

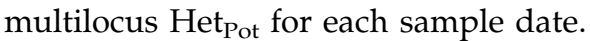

A complementary potence index for the common homozygote was also constructed:

$$
\operatorname{Com}_{\text {Pot }}=\left(P_{1}-P_{2}\right) /\left[2\left(\left|F-P_{2}\right|\right)\right]
$$

where $P_{1}, P_{2}$, and $F$ have the same values as above. Com $_{\text {Pot }}$ was normalized as above. If $\left(\left|F-P_{2}\right|\right)=0$, then

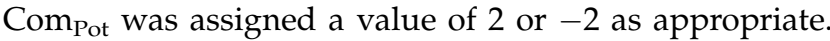
Interpretation of phenotypic interactions is slightly different. If $\mathrm{Com}_{\mathrm{Pot}}=0$, then the phenotypes of the homozygotes are the same. If $\operatorname{Com}_{\mathrm{Pot}}>0$ or $\mathrm{Com}_{\mathrm{Pot}}<0$, then the phenotype of the common homozygote is 
greater than or less than that of the uncommon homozygote, respectively. Further if $\mathrm{Com}_{\mathrm{Pot}}=1$ or -1 , the locus shows codominance. Otherwise, despite use of common parameters to compute Het $_{\text {Pot }}$ and Com Pot, $_{\text {, }}$ these indices are independent. That is, the performance of the heterozygote relative to the homozygotes does not affect the performance between homozygotes.

Allelic frequencies and Hardy-Weinberg equilibrium were computed by BIOSYS-1 (Swofford and Selander, 1989). Heterozygote deficiency was computed according to Selander (1970). Linkage disequilibrium was assessed indirectly by testing for independent association among genotypes for all possible pairs of loci using Fisher's exact tests because some dilocus genotypes were represented by fewer than five individuals. In order to account for the serial autocorrelation inherent in a timecourse experiment, the effect of environment and ontogeny on potence indices was tested in a mixed model analysis of covariance with single-locus potence index treated as a dependent variable, ontogeny treated as a fixed variable, and environment and time treated as random variables with time as a repeated measure to produce the appropriate denominator degrees of freedom. Multilocus potence indices were averaged among loci for each week during ontogeny and were treated statistically as above. For comparison, correlations between MLH and fresh weight were also computed for each sample date as discussed by Audo and Diehl (1995). Where appropriate, corrections for Type I errors were made using the sequential Bonferroni test (Rice, 1989). Figures were drawn with PSI-PLOT ${ }^{\circledR}$ for Windows ${ }^{\circledR}$ (Poly Software International Inc.). Statistics were computed with Proc Mixed, Proc GLM, and Proc FREQ of SAS ${ }^{\circledR} 8.2$ (c 1999-2001, SAS Institute Inc.).

\section{Results}

All loci in all environments were in Hardy-Weinberg equilibrium ( $\chi^{2}$ analysis; $\alpha=0.0083$ with sequential Bonferroni correction for lack of independence among loci within environments; Rice, 1989). There was no difference in genotype frequencies among environments at any locus ( $\chi^{2}$ analysis; $\alpha=0.0083$ ). Common allelic frequencies ranged from $0.54(\mathrm{Aap})$ to $0.77(\mathrm{Pgd})$. The mean heterozygote deficiency among loci was $0.017 \pm$ 0.034 (SE). The mean heterozygosity among loci and environments ranged from 0.313 to 0.609 . Because no individuals were added to or subtracted from the initial set of earthworms at any point during the analysis, allelic frequencies, heterozygote deficiencies, and heterozygosities were constant. Of the 15 possible pairs of loci in each environment, none showed significant deviations from independent association (Fisher's exact $\alpha=0.0033$ with sequential Bonferroni correction for lack of independence among 15 pairwise comparisons within environments; Rice, 1989). There was no significant effect of environment on either Het $_{\text {Pot }}$ or Com Pot $_{\text {potence index }}$ $Z=0.37-0.99$; Prob. $=0.160-0.356 ; \alpha=0.0083$ ) for any locus. Taken together, evidence suggests that earthworms distributed to different environments were drawn from a common population.

Figure 2 shows the effects of ontogeny on potence indices for both the heterozygote and the common homozygote for each locus averaged over environments. Two loci (Aap, Mdh) showed a significant increase in Het $_{\text {Pot }}$ with ontogeny $(\mathrm{F}=36.56,10.99$, respectively; $\mathrm{df}=1,50 ; P<0.0083$ with sequential Bonferroni corrections; Rice, 1989), and two loci (Pgd, Pgm) showed a

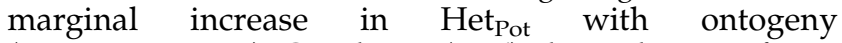
$(0.05>P>0.0083)$. One locus (Mpi) showed a significant decrease in Het $_{\text {Pot }}$ with ontogeny $(\mathrm{F}=12.41$; $\mathrm{df}=1,50$; $P<0.0083)$. Three loci (Aap, Gpd, Pgm) showed a significant decrease in Com Pot $_{\text {with ontogeny }}(\mathrm{F}=15.05$, 11.39, 14.39, respectively; $\mathrm{df}=1,50 ; P<0.0083$ with sequential Bonferroni corrections; Rice, 1989). There were few consistent patterns among individual loci. Several loci generally showed increases in Het $_{\mathrm{Pot}}$ or decreases in Com $_{\text {Pot }}$ with ontogeny, but these patterns differed with respect to the timing of the increase/ decrease and with respect to the specific phenotypic interactions that occurred.

Figure 3 shows the effect of ontogeny on both multilocus Het $_{\text {Pot }}$ and multilocus Com Pot $_{\text {averaged over }}$ environments. There was a significant increase in multi-

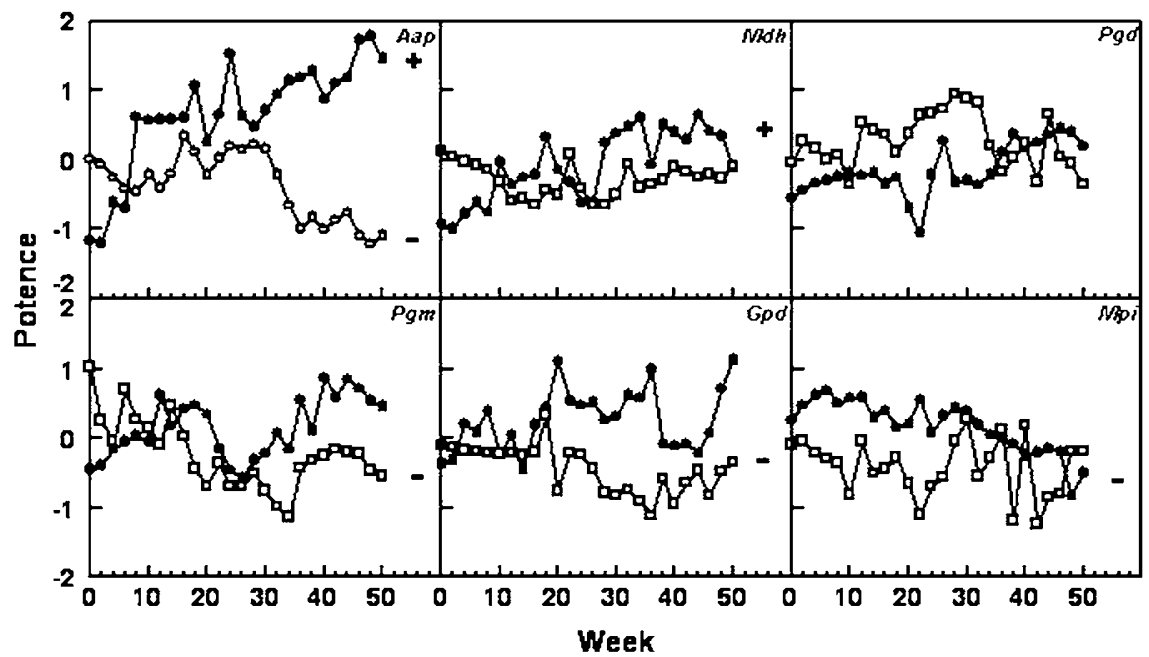

Figure 2 Heterozygosity potence (Het ${ }_{\text {Pot }}$, closed circles) and common homozygosity potence (Com ${ }_{\text {Pot }}$ open squares) for Aap, Mdh, Pgd, Pgm, $G p d$, and Mpi averaged over environments. $(+)$ and $(-)$ indicate significant $(P<0.0083)$ changes in potence with ontogeny. 


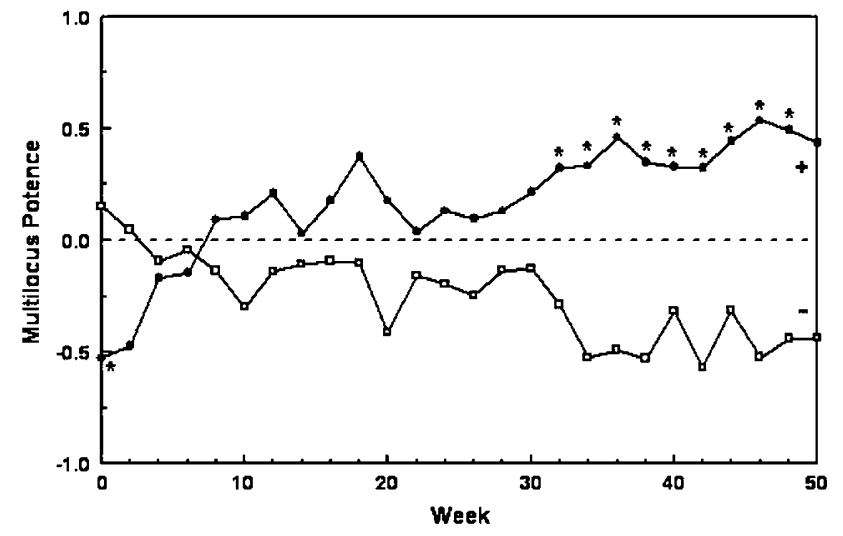

Figure $3 \mathrm{MLH}$ potence (closed circles) and multilocus common homozygosity potence (open squares) for six allozyme loci averaged over environments. $(+)$ and $(-)$ indicate significant $(P<0.05)$ changes in potence with ontogeny. Asterisks indicate times during ontogeny when a significant correlation $(P<0.05)$ between MLH and fresh weight could be detected for individuals pooled among environments.

locus Het Pot $_{\text {with ontogeny }}(\mathrm{F}=22.80 ; \mathrm{df}=1,50$; $P=0.0001)$ and a significant decrease in multilocus Com $_{\text {Pot }}$ with ontogeny $(\mathrm{F}=34.02 ; \mathrm{df}=1,50 ; P=0.0001)$ with no environment effect $(Z=0.94,0.98$, respectively; Prob. $=0.174,0.163)$. Within environments, MLH was only correlated positively with fresh weight for 2 weeks in one environment. For individuals pooled among environments, MLH was negatively correlated with fresh weight for week $0(P<0.05)$ and was positively correlated with fresh weight for weeks 32-48 $(P<0.05)$. Correlations were not adjusted with a sequential Bonferroni correction (Rice, 1989) because the point was to identify the time during ontogeny when such correlations were most likely to be detected. Generally, positive MLH-fresh weight relationships were detected when the mean multilocus Het $t_{\text {Pot }}$ approached 0.5.

\section{Discussion}

The potence index of Griffing (1990) was devised to assess the performance of quantitative traits in crossbred lineages compared to the respective inbred lineages, and the index provides a numerical value that could suggest a mechanism for heterosis. We have applied this index to assessing the performance of heterozygotes at single loci relative to the average performance of the respective homozygotes $\left(\right.$ Het $\left._{\mathrm{Pot}}\right)$. Also, we have constructed a comparable index ( $\mathrm{Com}_{\mathrm{Pot}}$ ) for assessing the performance of common homozygotes relative to uncommon

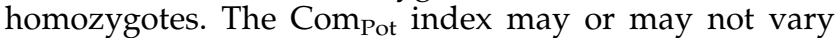

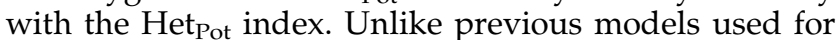
detecting heterozygosity-fitness relationships (Singh and Zouros, 1978; Smouse, 1986; Bush et al, 1987; David et al, 1995; David, 1998), the potence indices make few assumptions about relative performance among genotypes a priori. The model of Singh and Zouros (1978) assumes that the homozygous genotypes perform equally and that all loci have similar effects. The adaptive distance model of Smouse (1986) assumes that the performance of homozygous genotypes varies with allele frequency but that loci have similar effects. None of these assumptions is necessarily true, and our data suggest that they may often be false. Other models that rely on multiple regression approaches (Bush et al, 1987; David et al, 1995; David, 1998) make fewer assumptions but still cannot be rendered as a simple index. Instead of making broad assumptions about performance among genotypes, the potence indices use relative performance of genotypes obtained empirically to gain insight into potential phenotypic interactions and fitness relationships. The absence of assumptions increases the sensitivity of the index and reduces bias in determining the relationships between allozyme genotypes and fitness. Further, unlike some multiple regression models, the potence indices can be easily compared among genes, ontogenetic stages, and/or environments, and are easy to represent and interpret visually. These indices will only differ from measures of individual-based genotype performance as allele frequencies deviate greatly from 0.5 , but not necessarily so. Concordance of potence and MLH analyses (Figure 3) suggests that no such problem exists in this study.

The data show that ontogenetic factors can influence the association of fitness and allozyme genotypes. Although similarities exist, the ontogenetic patterns are not entirely consistent among the allozyme loci that we examined. Heterozygosity potence generally increases with ontogeny for three loci (Aap, Mdh, Pgd), but only one of these loci (Aap) shows consistent evidence of overdominance at any time during ontogeny. Common homozygote potence declines for three loci (Gpd, Pgm, Aap). This indicates that for these genes superior performance of the common homozygote early in ontogeny eventually gives way to superior performance of the heterozygote later in ontogeny. Only Aap shows an increase in heterozygosity potence, which coincides in part with a decrease in common homozygosity potence.

Many studies have shown inconsistencies in relative fitness of allozyme genotypes over time (reviewed by Mitton, 1997; David, 1998) and invoke selective mortality, migration, or spatial and/or temporal environmental variations as the causative agents that are responsible for the observed shifts in relative fitness. The design of this study excludes these factors as causative agents. Inconsistency can also be explained by inbreeding, which produces transitory relationships that may mimic overdominance when deleterious mutations are revealed in and purged from different regions of the genome between populations or generations. David et al (1995) suggested that multilocus heterosis, which seemed to occur early but not late in the life history of the bivalve Spisula ovalis, was caused by general inbreeding effects. Their study also used sequential growth measurements of the same individuals but did not compute heterosis for each stage in ontogeny per se. Thus, it is not clear if there was a change in the pattern of heterosis with ontogeny similar to the one reported here. In the present study, neither the presence of an ontogenetic pattern nor a difference in patterns among loci is compatible with the general effect of inbreeding, since allele frequencies and inbreeding coefficients are constant. This does not obviate the effect of inbreeding in other circumstances. However, examining relative genotypic performance using the same individuals through ontogeny or in different environments can be a very powerful experimental design for excluding the effects of inbreeding or other populational factors on such relationships. 
Although a general inbreeding effect can be eliminated as a causative agent here, none of these combinations of patterns is sufficient to determine whether heterosis or any other pattern is caused by allozyme loci directly or by loci tightly linked to them. On the one hand, the lack of any obvious functional association among loci showing similar ontogenetic patterns suggests that they are not directly responsible for that common pattern. If this is true, the absence of evidence of linkage among these loci either here or in a previous study (Diehl, 1988) suggests that there must be several target loci. On the other hand, a mechanism invoking linked target loci must accommodate the variety of specific patterns for potence of both heterozygotes and common homozygotes that exist among loci. For example, $M d h, P g d$, and Pgm show somewhat similar but modest increases in heterozygosity potence. Yet, magnitudes and patterns of changes in common homozygosity potence, and thus patterns of changes in apparent phenotypic interactions, differ among these loci. Aap shows a more complex pattern where heterozygosity potence increases after week 8 but common homozygote potence decreases only after week 32 . It is difficult to envision a simple, consistent linkage arrangement that can accommodate the variety of patterns observed in the loci tested here. Separating direct effect from local effect hypotheses has consistently been more problematic both practically and theoretically (Hansson and Westerberg, 2002) than separating either from general inbreeding effect hypotheses. Regardless, the assumption that loci contribute similarly to heterosis is falsified.

Heterozygotes at multiple loci appear to show a steady increase in average performance through ontogeny, while concurrently the common homozygotes show a steady decrease in average performance. Significant MLH-fresh weight relationships were only detected at the beginning of the life history (a negative correlation), and toward the end of the life history (positive correlations) for individuals pooled among environments. These patterns also differ from those studies that showed significant MLH-growth correlations early in ontogeny (about weeks 2-4) for this species (Audo and Diehl, 1995). The current patterns are compatible however with the selection reversal hypothesis of Gaffney et al (1990) to explain the frequent association of a heterozygote deficiency and heterosis in natural populations of marine bivalves. Because multilocus Het $_{\text {Pot }}$ represents an index averaged over loci showing different patterns, its value cannot imply a particular phenotypic interaction. That is, one cannot conclude that heterosis is caused by incomplete dominance even though the average value of multilocus Het $_{\text {Pot }}$ is nearly 0.5 after week 30 in ontogeny when positive MLH-fitness relationships can be detected. Populational and environmental factors may modify patterns of heterosis in natural populations, and general inbreeding effects may be responsible for or contribute to many cases of presumed heterosis, as described by McElroy and Diehl (2001). Nevertheless, the relationships seen here are more likely produced by the accumulation of changing phenotypic interactions coupled with differences in relative genotypic performance at multiple loci over the life history of an individual. Ontogeny may play an important role in heterosis. In natural populations, heterosis is likely the signature of several independent, but not mutually exclusive, mechanisms.

\section{Acknowledgements}

We thank Rebecca Brasfield, Melinda Chow, Warren Cox, April Heinsch, Carie Loundenslager, Tara Mann, Yonya Nabors, Theresa Roberts, and Lance Presley for assistance in data collection and earthworm husbandry. This research was supported by NSF Grant DEB9221094 to WJD.

\section{References}

Audo MC, Diehl WJ (1995). Effect of quantity and quality of environmental stress on multi locus heterozygosity-growth relationships in Eisenia fetida. Heredity 75: 98-105.

Booth CL, Woodruff DS, Gould SJ (1990). Lack of significant associations between allozyme heterozygosity and phenotypic traits in the land snail Cerion. Evolution 44: 210-213.

Britten HB (1996). Meta-analysis of the association between multilocus heterozygosity and fitness. Evolution 50: 2158-2164.

Bush RM, Smouse PE, Ledig FT (1987). The fitness consequences of multiple locus heterozygosity: the relationship between heterozygosity and growth rate in pitch pine Pinus rigida Mill. Evolution 41: 787-798.

Clayton JW, Tretiak DN (1972). Amine-citrate buffers for $\mathrm{pH}$ control in starch gel electrophoresis. J Fish Board Can 29: 1169-1172.

David P (1998). Heterozygosity-fitness correlations: new perspectives on old problems. Heredity 80: 531-537.

David P, Delay B, Berthou P, Jarne P (1995). Alternative models for allozyme-associated heterosis in the marine bivalve Sipsula ovalis. Genetics 139: 1719-1726.

David P, Jarne P (1997). Context-dependent survival differences among electrophoretic genotypes in natural populations of the marine bivalve Spisula ovalis. Genetics 146: 335-344.

Diehl WJ (1988). Genetics of carbohydrate metabolism and growth in Eisenia foetida (Oligochaeta: Lumbricidae). Heredity 61: 379-387.

Diehl WJ, Koehn RK (1985). Multiple-locus heterozygosity, mortality, and growth in a cohort of Mytilus edulis. Mar Biol 88: 265-271.

Diehl WJ, Williams DL (1992). Distribution of polymorphisms among pathways of carbohydrate metabolism in the earthworm Eisenia fetida (Oligochaeta). Comp Biochem Physiol 101B: 83-90.

Gaffney PM, Scott TM, Koehn RK, Diehl WJ (1990). Interrelationships of heterozygosity, growth rate and heterozygote deficiencies in the coot clam, Mulinia lateralis. Genetics 124: $687-699$.

Garton DW, Koehn RK, Scott TM (1984). Multiple-locus heterozygosity and the physiological energetics of growth in the coot clam, Mulinia lateralis, from a natural population. Genetics 108: 445-455.

Griffing B (1990). Use of a controlled-nutrient experiment to test heterosis hypotheses. Genetics 126: 753-767.

Hansson B, Westerberg L (2002). On the correlation between heterozygosity and fitness in natural populations. Mol Ecol 11: 2467-2474.

McElroy TC, Diehl WJ (2001). Heterosis in two closely related species of earthworm (Eisenia fetida and E. andrei). Heredity 87: 598-608.

McElroy TC, Presley ML, Diehl WJ (1999). Genotypes of multiple allozyme loci interact with an experimental environment to affect survivorship in earthworms (Eisenia andrei). Comp Biochem Phys 123A: 241-247.

Mitton JB (1997). Selection in Natural Populations. Oxford University Press: New York. 
Mulvey M, Keller GP, Meffe GK (1994). Single- and multiplelocus genotypes and life-history responses of Gambusia holbrooki reared at two temperatures. Evolution 48: 1810-1819.

Pasteur N, Pasteur G, Bonhomme R, Catalan J, Britton-Davidian J (1988). Practical Isozyme Genetics. John Wiley \& Sons: New York.

Pecon Slattery J, Lutz RA, Vrijenhoek RC (1993). Repeatability of correlations between heterozygosity, growth and survival in a natural population of the hard clam Mercenaria mercenaria L. J Exp Mar Biol Ecol 165: 209-224.

Presley ML, McElroy TC, Diehl WJ (1996). Soil moisture and temperature interact to affect growth, survivorship, fecundity, and fitness in the earthworm Eisenia fetida. Comp Biochem Physiol 114A: 319-326.

Rice WR (1989). Analyzing tables of statistical tests. Evolution 43: 223-225.

Selander RK (1970). Behavior and genetic variation in natural populations. Am Zool 10: 53-66.
Selander RK, Smith MH, Yang SY, Johnson WE, Gentry JB (1971). Biochemical polymorphism and systematics in the genus Peromyscus. I. Variation in the old-field mouse (Peromyscus polionotus). Stud Genet VI Univ Tex $\mathbf{7 1 0 3}$ 49-90.

Singh SM, Zouros E (1978). Genetic variation associated with growth rate in the American oyster (Crassostrea virginica). Evolution 32: 342-353.

Smouse PE (1986). The fitness consequences of multi-locus heterozygosity under the multiplicative overdominance and inbreeding depression models. Evolution 40: 946957.

Swofford DL, Selander RB (1989). BIOSYS-1. A Computer Program for the Analysis of Allelic Variation in Population Genetics and Biochemical Systematics Release 1.7. University of Illinois, Urbana, Illinois.

Whitlock M (1993). Lack of correlation between heterozygosity and fitness in forked fungus beetles. Heredity 70: 574-581. 